\title{
Difference Cordial Labeling of Subdivision of Snake Graphs
}

\author{
R. Ponraj ${ }^{1, *}$, S. Sathish Narayanan ${ }^{2}$, R. Kala ${ }^{3}$ \\ ${ }^{1}$ Department of Mathematics, Sri Paramakalyani College, Alwarkurichi-627412, India \\ ${ }^{2}$ Department of Mathematics, Thiruvalluvar College, Papanasam-627425, India \\ ${ }^{3}$ Department of Mathematics, Manonmaniam Sundaranar University, Tirunelveli-627012, India \\ *Corresponding Author: ponrajmaths@gmail.com
}

Copyright (C)2014 Horizon Research Publishing All rights reserved.

\begin{abstract}
Let $G$ be a $(p, q)$ graph. Let $f: V(G) \rightarrow\{1,2 \ldots, p\}$ be a function. For each edge $u v$, assign the label $|f(u)-f(v)| . \quad f$ is called a difference cordial labeling if $f$ is a one to one map and $\left|e_{f}(0)-e_{f}(1)\right| \leq 1$ where $e_{f}(1)$ and $e_{f}(0)$ denote the number of edges labeled with 1 and not labeled with 1 respectively. A graph with a difference cordial labeling is called a difference cordial graph. In this paper, we investigate the difference cordial labeling behavior of subdivision of some snake graphs.
\end{abstract}

Keywords Triangular snake, Quadrilateral snake, Subdivision, Double triangular snake, Double quadrilateral snake

\section{Introduction}

Let $G=(V, E)$ be a $(p, q)$ graph. In this paper we have considered only simple, non-trivial and undirected graphs. The number of vertices of $G$ is called the order of $G$ and the number of edges of $G$ is called the size $G$. The subdivision graph $S(G)$ of a graph $G$ is obtained by replacing each edge $u v$ by a path $u w v$. Graph labeling plays a vital role of numerous fields of sciences and some of them are astronomy, coding theory, x-ray crystallography, radar, database management etc.[1]. Ponrajet al. introduced difference cordial labeling of grpahs [3]. Let $G$ be a $(p, q)$ graph. Let $f: V(G) \rightarrow\{1,2 \ldots, p\}$ be a map. For each edge $u v$, assign the label $|f(u)-f(v)|$. $f$ is called a difference cordial labeling if $f$ is a one to one map and $\left|e_{f}(0)-e_{f}(1)\right| \leq 1$ where $e_{f}(1)$ and $e_{f}(0)$ denote the number of edges labeled with 1 and not labeled with 1 respectively. A graph with admits difference cordial labeling is called a difference cordial graph. In $[3,4,5,6]$ difference cordial labeling behavior of several graphs like path, cycle, complete graph, complete bipartite graph, bistar, wheel, web, $W_{n} \odot K_{2}$, $P_{m} \odot P_{n}$ and some more standard graphs have been investigated. In this paper, we investigate the difference cordial behaviour of $S\left(T_{n}\right), S\left(A\left(T_{n}\right)\right) S\left(D A\left(T_{n}\right)\right)$,
$S\left(Q_{n}\right), S\left(A\left(Q_{n}\right)\right), S\left(D A\left(Q_{n}\right)\right)$. Terms not defined here are used in the sense of Harary [2].

\section{Methodology}

In this article we use some number theory techniques for proving the main results.

\section{Main Results}

The triangular snake $T_{n}$ is obtained from the path $P_{n}$ by replacing each edge of the path by a triangle $C_{3}$. $S\left(T_{2}\right) \cong C_{6}$ and hence difference cordial [3].

Theorem $3.1 S\left(T_{n}\right)$ is difference cordial for all $n>2$.

Proof. Let $P_{n}$ be the path $u_{1} u_{2} \ldots u_{n}$. Let $V\left(T_{n}\right)=V\left(P_{n}\right) \cup\left\{v_{i}: 1 \leq i \leq n-1\right\}$. Let $V\left(S\left(T_{n}\right)\right)=$ $\left\{x_{i}, y_{i}, w_{i}: 1 \leq i \leq n-1\right\} \cup V\left(T_{n}\right)$ and $E\left(S\left(T_{n}\right)\right)=$ $\left\{u_{i} x_{i}, x_{i} v_{i}, y_{i} v_{i}, y_{i} u_{i+1}, u_{i} w_{i}, w_{i} u_{i+1}: 1 \leq i \leq n-1\right\}$. Define an injective map $f: \bar{V}\left(\bar{S}\left(T_{n}\right)\right) \rightarrow$ $\{1,2, \ldots, 5 n-4\}$ by

$$
\begin{array}{lll}
f\left(u_{i}\right) & =2 i-1 & 1 \leq i \leq n \\
f\left(w_{i}\right) & =2 i & 1 \leq i \leq n-1 \\
f\left(x_{i}\right) & =2 n+2 i-2 & 1 \leq i \leq n-1 \\
f\left(y_{i}\right) & =4 n-3+i & 1 \leq i \leq n-1 \\
f\left(v_{i}\right) & =2 n+2 i-1 & 1 \leq i \leq n-1 .
\end{array}
$$

Since $e_{f}(0)=e_{f}(1)=3 n-3, f$ is a difference cordial labeling of $S\left(T_{n}\right)$.

The Quadrilateral snake $Q_{n}$ is obtained from the path $P_{n}$ by replacing each edge of the path by a cycle $C_{4}$.

Theorem $3.2 S\left(Q_{n}\right)$ is difference cordial.

Proof. Let $P_{n}$ be the path $u_{1} u_{2} \ldots u_{n}$ and Let $V\left(Q_{n}\right)=$ $\left\{v_{i}, w_{i}: 1 \leq i \leq n-1\right\} \cup V\left(P_{n}\right)$. Let $V\left(S\left(Q_{n}\right)\right)=$ $\left\{x_{i}, u_{i}^{\prime}, z_{i}, y_{i}: 1 \leq i \leq n-1\right\} \quad \cup \quad V\left(Q_{n}\right) \quad$ and $E\left(S\left(Q_{n}\right)\right)=\left\{u_{i} u_{i}^{\prime}, u_{i}^{\prime} u_{i+1}, y_{i} u_{i+1}: 1 \leq i \leq n-1\right\} \cup$ 
$\left\{u_{i} x_{i}, x_{i} v_{i}, v_{i} z_{i}, z_{i} w_{i}, w_{i} y_{i}: 1 \leq i \leq n-1\right\}$. map $f: V\left(S\left(Q_{n}\right)\right) \rightarrow\{1,2, \ldots, 7 n-6\}$ by

$$
\begin{aligned}
f\left(u_{i}\right) & =2 i-1 & & 1 \leq i \leq n \\
f\left(u_{i}^{\prime}\right) & =2 i & & 1 \leq i \leq n-1 \\
f\left(v_{i}\right) & =2 n+3 i-3 & & 1 \leq i \leq n-1 \\
f\left(z_{i}\right) & =2 n+3 i-2 & & 1 \leq i \leq n-1 \\
f\left(w_{i}\right) & =2 n+3 i-1 & & 1 \leq i \leq n-1 \\
f\left(x_{i}\right) & =5 n-4+i & & 1 \leq i \leq n-1 \\
f\left(y_{i}\right) & =6 n-5+i & & 1 \leq i \leq n-1 .
\end{aligned}
$$

Since $e_{f}(0)=e_{f}(1)=4 n-4, f$ is a difference cordial labeling of $S\left(Q_{n}\right)$.

A double triangular snake $D T_{n}$ consists of two triangular snakes that have a common path.

Theorem 3.3 $S\left(D T_{n}\right)$ is difference cordial.

Proof. Let $V\left(S\left(D T_{n}\right)\right)=\left\{u_{i}: 1 \leq i \leq n\right\} \cup$ $\left\{x_{i}, y_{i}, v_{i}, x_{i}^{\prime}, y_{i}^{\prime}, w_{i}, z_{i}: 1 \leq i \leq n-1\right\}$ and $E\left(S\left(D T_{n}\right)\right)$ $=\left\{u_{i} z_{i}, z_{i} u_{i+1}, u_{i} x_{i}, y_{i} u_{i+1}, x_{i} v_{i}, u_{i} x_{i}^{\prime}: 1 \leq i \leq n-1\right\} \cup$ $\left\{v_{i} y_{i}, w_{i} y_{i}^{\prime}, x_{i}^{\prime} w_{i}, y_{i}^{\prime} u_{i+1}: 1 \leq i \leq n-1\right\}$. Define a map $f: V\left(S\left(D T_{n}\right)\right) \rightarrow\{1,2, \ldots, 8 n-7\}$ by

$$
\begin{array}{rlrl}
f\left(u_{i}\right) & =4 i-3 & & 1 \leq i \leq n \\
f\left(z_{i}\right) & =7 n-6+i & & 1 \leq i \leq n-1 \\
f\left(x_{i}\right) & =4 i-2 & & 1 \leq i \leq n-1 \\
f\left(v_{i}\right) & =4 i-1 & & 1 \leq i \leq n-1 \\
f\left(y_{i}\right) & =4 i & 1 \leq i \leq n-1 \\
f\left(x_{i}^{\prime}\right) & =4 n+2 i-4 & 1 \leq i \leq n-1 \\
f\left(w_{i}\right) & =4 n+2 i-3 & 1 \leq i \leq n-1 \\
f\left(y_{i}^{\prime}\right) & =6 n-5+i & 1 \leq i \leq n-1 .
\end{array}
$$

Obviously the above vertex labeling is a difference cordial labeling of $S\left(D T_{n}\right)$.

A double quadrilateral snake $D Q_{n}$ consists of two triangular snakes that have a common path.

Theorem 3.4 $S\left(D Q_{n}\right)$ is difference cordial.

Proof. Let $V\left(S\left(D Q_{n}\right)\right)=V\left(D Q_{n}\right) \cup$ $\left\{u_{i}^{\prime}, v_{i}^{\prime}, w_{i}^{\prime}, z_{i}^{\prime}, x_{i}^{\prime}, y_{i}^{\prime}, z_{i}: 1 \leq i \leq n-1\right\} \quad$ and $E\left(S\left(D Q_{n}\right)\right)=\left\{u_{i} u_{i}^{\prime}, u_{i}^{\prime} u_{i+1}: 1 \leq i \leq n-1\right\} \cup$ $\left\{u_{i} v_{i}^{\prime}, u_{i} x_{i}^{\prime}, v_{i}^{\prime} v_{i}, x_{i}^{\prime} x_{i}, v_{i} z_{i}^{\prime}, x_{i} z_{i}, z_{i}^{\prime} w_{i}: 1 \leq i \leq n-1\right\} \cup$ $\left\{z_{i} y_{i}, w_{i} w_{i}^{\prime}, y_{i} y_{i}^{\prime}, w_{i}^{\prime} u_{i+1}, y_{i}^{\prime} u_{i+1}: 1 \leq i \leq n-1\right\}$. Define a map $f: V\left(S\left(D Q_{n}\right)\right) \rightarrow\{1,2, \ldots, 12 n-11\}$

$$
\begin{array}{rlrl}
f\left(u_{i}\right) & =6 i-5 & & 1 \leq i \leq n \\
f\left(v_{i}^{\prime}\right) & =6 i-4 & & 1 \leq i \leq n-1 \\
f\left(v_{i}\right) & =6 i-3 & & 1 \leq i \leq n-1 \\
f\left(z_{i}^{\prime}\right) & =6 i-2 & & 1 \leq i \leq n-1 \\
f\left(w_{i}\right) & =6 i-1 & & 1 \leq i \leq n-1 \\
f\left(w_{i}^{\prime}\right) & =6 i & & 1 \leq i \leq n-1 \\
f\left(x_{i}^{\prime}\right) & =6 n+2 i-6 & & 1 \leq i \leq n-1 \\
f\left(x_{i}\right) & =6 n+2 i-5 & & 1 \leq i \leq n-1 \\
f\left(z_{i}\right) & =8 n+i-7 & & 1 \leq i \leq n-1 \\
f\left(y_{i}\right) & =9 n+i-8 & & 1 \leq i \leq n-1 \\
f\left(y_{i}^{\prime}\right) & =10 n+i-9 & & 1 \leq i \leq n-1 \\
f\left(u_{i}^{\prime}\right) & =11 n+i-10 & 1 \leq i \leq n-1 .
\end{array}
$$

Since $e_{f}(0)=e_{f}(1)=7 n-7, f$ is a difference cordial labeling of $S\left(D Q_{n}\right)$.

An alternate triangular snake $A\left(T_{n}\right)$ is obtained from a path $u_{1} u_{2} \ldots, u_{n}$ by joining $u_{i}$ and $u_{i+1}$ (alternatively) to new vertex $v_{i}$. That is every alternate edge of a path is replaced by $C_{3}$.

Theorem 3.5 $S\left(A\left(T_{n}\right)\right)$ is difference cordial.

Proof. Let the edges $u_{i} u_{i+1}, u_{i} v_{i}$ and $v_{i} u_{i+1}$ be subdivided by $u_{i}^{\prime}, x_{i}$ and $y_{i}$ respectively.

Case 1. Let the triangle be starts from $u_{1}$ and ends with $u_{n}$.

In this case $S\left(A\left(T_{n}\right)\right)$ consists of $\frac{7 n-2}{2}$ vertices and $4 n-2$ edges. Define a map $f: V\left(S\left(A\left(T_{n}\right)\right)\right) \rightarrow$ $\left\{1,2, \ldots, \frac{7 n-2}{2}\right\}$ by

$$
\begin{aligned}
f\left(u_{i}\right) & =2 i-1 & & 1 \leq i \leq n \\
f\left(u_{i}^{\prime}\right) & =2 i & & 1 \leq i \leq n-1 \\
f\left(x_{i}\right) & =2 n-1+i & & 1 \leq i \leq \frac{n}{2} \\
f\left(y_{i}\right) & =\frac{5 n-2}{2}+i & & 1 \leq i \leq \frac{n}{2} \\
f\left(v_{\frac{n}{2}-i+1}\right) & =3 n-1+i & & 1 \leq i \leq \frac{n}{2} .
\end{aligned}
$$

Since $e_{f}(0)=e_{f}(1)=2 n-1, f$ is a difference cordial labeling of $S\left(A\left(T_{n}\right)\right)$.

Case 2. Let the triangle be starts from $u_{2}$ and ends with $u_{n-1}$.

In this case, $S\left(A\left(T_{n}\right)\right)$ has $\frac{7 n-8}{2}$ vertices and $4 n-6$ edges. Define an injective map $f: V\left(S\left(A\left(T_{n}\right)\right)\right) \rightarrow$ $\left\{1,2, \ldots, \frac{7 n-8}{2}\right\}$ by

$$
\begin{aligned}
f\left(u_{i}\right) & =2 i-2 & & 2 \leq i \leq n \\
f\left(u_{i}^{\prime}\right) & =2 i-1 & & 1 \leq i \leq n-1 \\
f\left(x_{i}\right) & =2 n-2+i & & 1 \leq i \leq \frac{n-2}{2} \\
f\left(y_{i}\right) & =\frac{5 n-6}{2}+i & & 1 \leq i \leq \frac{n-2}{2} \\
f\left(v_{i}\right) & =3 n-3+i & & 1 \leq i \leq \frac{n-2}{2} .
\end{aligned}
$$


$f\left(u_{1}\right)=3 n-3$. Obviously, $f$ is a difference cordial labeling of $S\left(A\left(T_{n}\right)\right)$.

Case 3. Let the triangle be starts from $u_{2}$ and ends with $u_{n}$.

In this case, the order and size of $S\left(A\left(T_{n}\right)\right)$ are $\frac{7 n-5}{2}$ and $4 n-4$ respectively. The difference cordial labeling of $S\left(A\left(T_{3}\right)\right)$ is given in figure 1 .

$\begin{array}{ll}7 & \\ & 6\end{array}$

$\begin{array}{lllll}8 & 1 & 2 & 3 & 4\end{array}$

Figure 1

For $n>3$, Define an injective map $f: V\left(S\left(A\left(T_{n}\right)\right)\right) \rightarrow$ $\left\{1,2, \ldots, \frac{7 n-5}{2}\right\}$ by

$$
\begin{aligned}
f\left(u_{i}\right) & =2 i-1 & & 1 \leq i \leq n \\
f\left(u_{i}^{\prime}\right) & =2 i & & 1 \leq i \leq n-1 \\
f\left(x_{i}\right) & =2 n-1+i & & 1 \leq i \leq \frac{n-1}{2} \\
f\left(y_{i}\right) & =\frac{5 n-3}{2}+i & & 1 \leq i \leq \frac{n-1}{2} \\
f\left(v_{i}\right) & =3 n-2+i & & 1 \leq i \leq \frac{n-1}{2} .
\end{aligned}
$$

Since $e_{f}(0)=e_{f}(1)=2 n-2, f$ is a difference cordial labeling of $S\left(A\left(T_{n}\right)\right)$.

A double alternate triangular snake $D A\left(T_{n}\right)$ consist of two triangular snakes that have a common path. That is, a double alternate triangular snake is obtained from a path $u_{1} u_{2} \ldots u_{n}$ by joining $u_{i}$ and $u_{i+1}$ (alternatively) to two new vertices $v_{i}$ and $w_{i}$.

Theorem 3.6 $S\left(D A\left(T_{n}\right)\right)$ is difference cordial.

Proof. Let the edges $u_{i} u_{i+1}, u_{i} v_{i}, v_{i} u_{i+1}, u_{i} w_{i}, w_{i} u_{i+1}$ be subdivided by $u_{i}^{\prime}, x_{i}, y_{i}, x_{i}^{\prime}, y_{i}^{\prime}$ respectively.

Case 1. Let the two triangles be starts from $u_{1}$ and ends with $u_{n}$.

Here, the number of vertices and edges in $S\left(D A\left(T_{n}\right)\right)$ are $5 n-1$ and $6 n-2$ respectively. Define a map $f$ : $V\left(S\left(D A\left(T_{n}\right)\right)\right) \rightarrow\{1,2, \ldots, 5 n-1\}$ by

$$
\begin{aligned}
f\left(u_{i}\right) & =2 i-1 & & 1 \leq i \leq n \\
f\left(u_{i}^{\prime}\right) & =2 i & & 1 \leq i \leq n-1 \\
f\left(x_{i}\right) & =2 n-2+2 i & & 1 \leq i \leq \frac{n}{2} \\
f\left(v_{i}\right) & =2 n-1+2 i & & 1 \leq i \leq \frac{n}{2} \\
f\left(y_{\frac{n}{2}-i+1}\right) & =3 n-1+i & & 1 \leq i \leq \frac{n}{2} \\
f\left(x_{i}^{\prime}\right) & =\frac{7 n-2}{2}+i & & 1 \leq i \leq \frac{n}{2} \\
f\left(y_{i}^{\prime}\right) & =4 n-1+i & & 1 \leq i \leq \frac{n}{2} \\
f\left(w_{i}\right) & =\frac{9 n-2}{2}+i & & 1 \leq i \leq \frac{n}{2} .
\end{aligned}
$$

Since $e_{f}(0)=e_{f}(1)=3 n-1, f$ is a difference cordial labeling of $S\left(D A\left(T_{n}\right)\right)$.

Case 2. Let the two triangles be starts from $u_{2}$ and ends with $u_{n-1}$.
In this case, the order and size of $S\left(D A\left(T_{n}\right)\right)$ are $5 n-7$ and $6 n-10$ respectively. Define a function $f$ : $V\left(S\left(D A\left(T_{n}\right)\right)\right) \rightarrow\{1,2, \ldots, 5 n-7\}$ by $f\left(u_{1}\right)=2 n-1$,

$$
\begin{aligned}
f\left(u_{i}\right) & =2 i-2 & & 2 \leq i \leq n \\
f\left(u_{i}^{\prime}\right) & =2 i-1 & & 1 \leq i \leq n-1 \\
f\left(x_{i}\right) & =2 n-2+2 i & & 1 \leq i \leq \frac{n-2}{2} \\
f\left(v_{i}\right) & =2 n-1+2 i & & 1 \leq i \leq \frac{n-2}{2} \\
f\left(y_{i}\right) & =4 n-5+i & & 1 \leq i \leq \frac{n-2}{2} \\
f\left(x_{i}^{\prime}\right) & =3 n+2 i-4 & & 1 \leq i \leq \frac{n-2}{2} \\
f\left(y_{i}^{\prime}\right) & =\frac{9 n-12}{2}+i & & 1 \leq i \leq \frac{n-2}{2} \\
f\left(w_{i}\right) & =3 n+2 i-3 & & 1 \leq i \leq \frac{n-2}{2} .
\end{aligned}
$$

Since $e_{f}(0)=e_{f}(1)=3 n-5, f$ is a difference cordial labeling of $S\left(D A\left(T_{n}\right)\right)$.

Case 3. Let the two triangles be starts from $u_{2}$ and ends with $u_{n}$.

In this case, the order and size of $S\left(D A\left(T_{n}\right)\right)$ consists of $5 n-4$ vertices and $6 n-6$ edges respectively. Define a function $f: V\left(S\left(D A\left(T_{n}\right)\right)\right) \rightarrow\{1,2, \ldots, 5 n-4\}$ by

$$
\begin{aligned}
f\left(u_{i}\right) & =2 i-1 & & 1 \leq i \leq n \\
f\left(u_{i}^{\prime}\right) & =2 i & & 1 \leq i \leq n-1 \\
f\left(x_{i}\right) & =2 n+2 i-2 & & 1 \leq i \leq \frac{n-1}{2} \\
f\left(v_{i}\right) & =2 n+2 i-1 & & 1 \leq i \leq \frac{n-1}{2} \\
f\left(x_{i}^{\prime}\right) & =3 n+2 i-3 & & 1 \leq i \leq \frac{n-1}{2} \\
f\left(w_{i}\right) & =3 n+2 i-2 & & 1 \leq i \leq \frac{n-1}{2} \\
f\left(y_{i}\right) & =4 n-3+i & & 1 \leq i \leq \frac{n-1}{2} \\
f\left(y_{i}^{\prime}\right) & =\frac{9 n-7}{2}+i & & 1 \leq i \leq \frac{n-1}{2} .
\end{aligned}
$$

Since $e_{f}(0)=e_{f}(1)=3 n-3, f$ is a difference cordial labeling of $S\left(D A\left(T_{n}\right)\right)$.

An alternate quadrilateral snake $A\left(Q_{n}\right)$ is obtained from a path $u_{1} u_{2} \ldots u_{n}$ by joining $u_{i}, u_{i+1}$ (alternatively) to new vertices $v_{i}, w_{i}$ respectively and then joining $v_{i}$ and $w_{i}$. That is every alternate edge of a path is replaced by a cycle $C_{4}$.

Theorem 3.7 $S\left(A Q_{n}\right)$ is difference cordial.

Proof. Let the edges $u_{i} u_{i+1}, u_{i} v_{i}, v_{i} w_{i}, w_{i} u_{i+1}$ be subdivided by $u_{i}^{\prime}, v_{i}^{\prime}, z_{i}, w_{i}^{\prime}$ respectively.

Case 1. Let the squares be starts from $u_{1}$ and ends with $u_{n}$.

In this case the order and size of $S\left(A Q_{n}\right)$ are $\frac{9 n-2}{2}$ and $5 n-2$ respectively. Define a map $f: V\left(S\left(A Q_{n}\right)\right) \rightarrow$ 
$\left\{1,2, \ldots, \frac{9 n-2}{2}\right\}$ as follows:

$$
\begin{aligned}
f\left(u_{i}\right) & =2 i-1 & & 1 \leq i \leq n \\
f\left(u_{i}^{\prime}\right) & =2 i & & 1 \leq i \leq n-1 \\
f\left(v_{i}^{\prime}\right) & =2 n+2 i-2 & & 1 \leq i \leq \frac{n}{2} \\
f\left(v_{i}\right) & =2 n+2 i-1 & & 1 \leq i \leq \frac{n}{2} \\
f\left(w_{i}^{\prime}\right) & =3 n-1+i & & 1 \leq i \leq \frac{n}{2} \\
f\left(z_{i}\right) & =\frac{7 n-2}{2}+i & & 1 \leq i \leq \frac{n}{2} \\
f\left(w_{\frac{n}{2}-i+1}\right) & =4 n-1+i & & 1 \leq i \leq \frac{n}{2} .
\end{aligned}
$$

Since $e_{f}(0)=e_{f}(1)=\frac{5 n-2}{2}, f$ is a difference cordial labeling of $S\left(A Q_{n}\right)$.

Case 2. Let the squares be starts from $u_{2}$ and ends with $u_{n-1}$.

The difference cordial labeling of $S\left(A Q_{4}\right)$ is given in figure 2 .

$$
\begin{array}{lll}
10 & 12 & 11
\end{array}
$$

$$
8
$$

$\begin{array}{lllllll}7 & 1 & 2 & 3 & 4 & 5 & 6\end{array}$

Figure 2

For $n>4$, Define a map $f: V\left(S\left(A Q_{n}\right)\right) \rightarrow$ $\left\{1,2, \ldots, \frac{9 n-12}{2}\right\}$ by

$$
\begin{aligned}
f\left(u_{i}\right) & =2 i-2 & & 2 \leq i \leq n \\
f\left(u_{i}^{\prime}\right) & =2 i-1 & & 1 \leq i \leq n-1 \\
f\left(v_{i}^{\prime}\right) & =2 n+2 i-3 & & 1 \leq i \leq \frac{n-2}{2} \\
f\left(v_{i}\right) & =2 n+2 i-2 & & 1 \leq i \leq \frac{n-2}{2} \\
f\left(w_{i}^{\prime}\right) & =3 n-4+i & & 1 \leq i \leq \frac{n-2}{2} \\
f\left(z_{i}\right) & =\frac{7 n-10}{2}+i & & 1 \leq i \leq \frac{n-2}{2} \\
f\left(w_{i}\right) & =4 n-6+i & & 1 \leq i \leq \frac{n-2}{2}
\end{aligned}
$$

and $f\left(u_{1}\right)=\frac{9 n-12}{2}$. Since $e_{f}(0)=e_{f}(1)=5 n-8, f$ is a difference cordial labeling of $S\left(A Q_{n}\right)$.

Case 3. Let the squares be starts from $u_{2}$ and ends with $u_{n}$.

The difference cordial labeling of $S\left(A Q_{3}\right)$ is given in figure 3 .

$\begin{array}{lll}8 & 9 & 7 \\ 6 & & \\ & & \\ 3 & 4 & 5\end{array}$

Figure 3
For $n>3$, Define a map $f: V\left(S\left(A Q_{n}\right)\right) \rightarrow$ $\left\{1,2, \ldots, \frac{9 n-7}{2}\right\}$ by

$$
\begin{aligned}
f\left(u_{i}\right) & =2 i-1 & & 1 \leq i \leq n \\
f\left(u_{i}^{\prime}\right) & =2 i & & 1 \leq i \leq n-1 \\
f\left(v_{i}^{\prime}\right) & =2 n+2 i-2 & & 1 \leq i \leq \frac{n-1}{2} \\
f\left(v_{i}\right) & =2 n+2 i-1 & & 1 \leq i \leq \frac{n-1}{2} \\
f\left(z_{i}\right) & =3 n-2+i & & 1 \leq i \leq \frac{n-1}{2} \\
f\left(w_{i}\right) & =\frac{7 n-5}{2}+i & & 1 \leq i \leq \frac{n-1}{2} \\
f\left(w_{i}^{\prime}\right) & =4 n-3+i & & 1 \leq i \leq \frac{n-1}{2} .
\end{aligned}
$$

Since $e_{f}(0)=e_{f}(1)=\frac{5 n-5}{2}, f$ is a difference cordial labeling of $S\left(A Q_{n}\right)$.

Illustration 1 The difference cordial labeling of $D A\left(Q_{8}\right)$ is given in figure 4.

$\begin{array}{llllllllllll}17 & 28 & 35 & 19 & 29 & 34 & 21 & 30 & 33 & 23 & 31 & 32\end{array}$

\begin{tabular}{lllllllllllllllll}
16 & \multicolumn{1}{r}{} & 24 & 18 & \multicolumn{1}{c}{25} & 20 & & 26 & 22 & & 27 \\
1 & 2 & 3 & 4 & 5 & 6 & 7 & 8 & 9 & 10 & 11 & 12 & 13 & 14 & 15
\end{tabular}

Figure 4

The difference cordial labeling of $D A\left(Q_{8}\right)$ is given in figure 5 .

$\begin{array}{lllllllll}16 & 24 & 27 & 18 & 25 & 28 & 20 & 26 & 29\end{array}$

\begin{tabular}{llllllllllllllll} 
& 15 & \multicolumn{1}{c}{21} & 17 & \multicolumn{1}{c}{22} & 19 & \multicolumn{2}{c}{23} & \\
30 & 1 & 2 & 3 & 4 & 5 & 6 & 7 & 8 & 9 & 10 & 11 & 12 & 13 & 14
\end{tabular}

Figure 5

The difference cordial labeling of $D A\left(Q_{7}\right)$ is given in figure 6 .

$$
\begin{array}{lllllllll}
15 & 20 & 23 & 17 & 21 & 24 & 19 & 22 & 25
\end{array}
$$

$\begin{array}{llllll}14 & 26 & 16 & 27 & 18 & 28\end{array}$

$\begin{array}{lllllllllllll}1 & 2 & 3 & 4 & 5 & 6 & 7 & 8 & 9 & 10 & 11 & 12 & 13\end{array}$

Figure 6

A double alternate quadrilateral snake $D A\left(Q_{n}\right)$ consists of two alternate quadrilateral snakes that have a common path. That is, a double alternate quadrilateral snake is obtained from a path $u_{1} u_{2} \ldots u_{n}$ by joining $u_{i}$ and $u_{i+1}$ (alternatively) to new vertices $v_{i}, x_{i}$ and $w_{i}$, $y_{i}$ respectively and then joining $v_{i}, w_{i}$ and $x_{i}, y_{i}$.

Theorem 3.8 $S\left(D A Q_{n}\right)$ is difference cordial.

Proof. Let the egdes $u_{i} u_{i+1}, u_{i} v_{i}, v_{i} w_{i}, w_{i} u_{i+1}, u_{i} x_{i}$, $x_{i} y_{i}, y_{i} u_{i+1}$ be subdivided by $u_{i}^{\prime}, v_{i}^{\prime}, z_{i}, w_{i}^{\prime}, x_{i}^{\prime}, z_{i}^{\prime}, y_{i}^{\prime}$ respectively.

Case 1. Let the squares be starts from $u_{1}$ and ends with $u_{n}$.

The order and size of $S\left(D A Q_{n}\right)$ are $7 n-1$ and $8 n-2$ 
respectively. Define a map $f: V\left(S\left(D A Q_{n}\right)\right) \rightarrow$ $\{1,2, \ldots, 7 n-1\}$ by

$$
\begin{array}{rlrl}
f\left(u_{i}\right) & =2 i-1 & & 1 \leq i \leq n \\
f\left(u_{i}^{\prime}\right) & =2 i & & 1 \leq i \leq n-1 \\
f\left(v_{i}\right) & =2 n+3 i-3 & & 1 \leq i \leq \frac{n}{2} \\
f\left(z_{i}\right) & =2 n+3 i-2 & & 1 \leq i \leq \frac{n}{2} \\
f\left(w_{i}\right) & =2 n+3 i-1 & & 1 \leq i \leq \frac{n}{2} \\
f\left(x_{i}\right) & =\frac{7 n-6}{2}+3 i & & 1 \leq i \leq \frac{n}{2} \\
f\left(z_{i}^{\prime}\right) & =\frac{7 n-4}{2}+3 i & & 1 \leq i \leq \frac{n}{2} \\
f\left(y_{i}\right) & =\frac{7 n-2}{2}+3 i & & 1 \leq i \leq \frac{n}{2} \\
f\left(x_{i}^{\prime}\right) & =\frac{11 n-2}{2}+i & & 1 \leq i \leq \frac{n}{2} \\
f\left(w_{i}^{\prime}\right) & =\frac{13 n-2}{2}+i & & 1 \leq i \leq \frac{n}{2} \\
f\left(y_{\frac{n}{2}}^{\prime}-i+1\right) & =\frac{5 n-1+i}{f\left(v_{i}^{\prime}\right)}= & & 1 \leq i \leq \frac{n}{2} \\
& =15-1+i & 1 \leq i \leq \frac{n}{2} .
\end{array}
$$

Since $e_{f}(0)=e_{f}(1)=4 n-1, f$ is a difference cordial labeling of $S\left(D A Q_{n}\right)$.

Case 2. Let the squares be starts from $u_{2}$ and ends with $u_{n-1}$.

The order and size of $S\left(D A Q_{n}\right)$ are $7 n-11$ and $8 n-14$ respectively. Define a map $f: V\left(S\left(D A Q_{n}\right)\right) \rightarrow$ $\{1,2, \ldots, 7 n-11\}$ by

$$
\begin{array}{rlrl}
f\left(u_{i}\right) & =2 i-2 & & 2 \leq i \leq n \\
f\left(u_{i}^{\prime}\right) & =2 i-1 & & 1 \leq i \leq n-1 \\
f\left(v_{i}\right) & =2 n+3 i-4 & & 1 \leq i \leq \frac{n-2}{2} \\
f\left(z_{i}\right) & =2 n+3 i-3 & & 1 \leq i \leq \frac{n-2}{2} \\
f\left(w_{i}\right) & =2 n+3 i-2 & & 1 \leq i \leq \frac{n-2}{2} \\
f\left(x_{i}\right) & =\frac{7 n-14}{2}+3 i & & 1 \leq i \leq \frac{n-2}{2} \\
f\left(z_{i}^{\prime}\right) & =\frac{7 n-12}{2}+3 i & & 1 \leq i \leq \frac{n-2}{2} \\
f\left(y_{i}\right) & =\frac{7 n-10}{2}+3 i & & 1 \leq i \leq \frac{n-2}{2} \\
f\left(w_{i}^{\prime}\right) & =\frac{11 n-18}{2}+i & & 1 \leq i \leq \frac{n-2}{2} \\
f\left(y_{i}^{\prime}\right) & =\frac{13 n-22}{2}+i & & 1 \leq i \leq \frac{n-2}{2} \\
f\left(v_{i}^{\prime}\right) & =5 n-8+i & & 1 \leq i \leq \frac{n-2}{2} \\
f\left(x_{i}^{\prime}\right) & =6 n-10+i & 1 \leq i \leq \frac{n-2}{2}
\end{array}
$$

and $f\left(u_{1}\right)=7 n-11$. Since $e_{f}(0)=e_{f}(1)=4 n-7, f$ is a difference cordial labeling of $S\left(D A Q_{n}\right)$.

Case 3. Let the squares be starts from $u_{2}$ and ends with $u_{n}$.

Assign the labels to the vertices $u_{i} \quad(1 \leq i \leq n)$, $u_{i}^{\prime}(1 \leq i \leq n-1), v_{i}, z_{i}, w_{i}\left(1 \leq i \leq \frac{n-1}{2}\right)$ as in case 1 and define

$$
\begin{aligned}
f\left(x_{i}\right) & =\frac{7 n-9}{2}+3 i & & 1 \leq i \leq \frac{n-1}{2} \\
f\left(z_{i}^{\prime}\right) & =\frac{7 n-7}{2}+3 i & & 1 \leq i \leq \frac{n-1}{2} \\
f\left(y_{i}\right) & =\frac{7 n-5}{2}+3 i & & 1 \leq i \leq \frac{n-1}{2} \\
f\left(w_{i}^{\prime}\right) & =\frac{11 n-9}{2}+i & & 1 \leq i \leq \frac{n-1}{2} \\
f\left(y_{i}^{\prime}\right) & =\frac{13 n-11}{2}+i & & 1 \leq i \leq \frac{n-1}{2} \\
f\left(v_{i}^{\prime}\right) & =5 n-4+i & & 1 \leq i \leq \frac{n-1}{2} \\
f\left(x_{i}^{\prime}\right) & =6 n-5+i & & 1 \leq i \leq \frac{n-1}{2} .
\end{aligned}
$$

Since $e_{f}(0)=e_{f}(1)=4 n-4, f$ is a difference cordial labeling of $S\left(D A Q_{n}\right)$.

\section{Conclusion}

In this paper we have proved that subdivision of quadrilateral snakes, triangular snakes, Alternate triangular snakes, double triangular snakes, Alternate quadrilateral snakes, Double quadrilateral snakes, Double alternate triangular snakes, double alternate quadrilateral snakes are difference cordial. Investigation of difference cordial labeling behavior of subdivision of $G_{1} \cup G_{2}, G_{1}+G_{2}, G_{1} \times G_{2}$ etc are the open problems for future research.

\section{Acknowledgement}

The authors thank the refree for his valuable suggestions and comments.

\section{REFERENCES}

[1] J. A. Gallian, A Dynamic survey of graph labeling, The Electronic Journal of Combinatorics, 18 (2012) \#Ds6.

[2] F. Harary, Graph theory, Addision wesley, New Delhi (1969).

[3] R. Ponraj, S. Sathish Narayanan and R. Kala, Difference cordial labeling of graphs, Global Journal of Mathematical Sicences: Theory and Practical, 5(2013), 185-196.

[4] R. Ponraj, S. Sathish Narayanan and R. Kala, Difference cordial labeling of graphs obtained from double snakes, International Journal of Mathematics Research, 5,Number3 (2013), 317-322.

[5] R. Ponraj and S. Sathish Narayanan, Difference cordiality of some graphs obtained from double alternate snake graphs, Global Journal of Mathematical Sciences, 5(2013), 167-175. 
[6] R. Ponraj, S. Sathish Narayanan and R. Kala, Differput. Sci., 3(2013), 1237-1251. ence cordial labeling of corona graphs, J. Math. Com- 